\title{
Tools for Stability of Switching Linear Systems: Gain Automata and Delay Compensation
}

\author{
Rom Langerak \\ Department of Computer Science \\ University of Twente \\ P.O.Box 217 \\ 7500 AE Enschede, The Netherlands \\ langerak@cs.utwente.nl
}

\author{
Jan Willem Polderman \\ Department of Applied Mathematics \\ University of Twente \\ P.O.Box 217 \\ 7500 AE Enschede, The Netherlands \\ J.W.Polderman@math. utwente.nl
}

\begin{abstract}
The topic of this paper is the analysis of stability for a class of switched linear systems, modeled by hybrid automata. In each location of the hybrid automaton the dynamics is assumed to be linear and asymptotically stable; the guards on the transitions are hyperplanes in the state space. For each location an estimate is made of the gain via a Lyapunov function for the dynamics in that location, given a pair of ingoing and outgoing transitions. It is shown how to obtain the best possible estimate by optimizing the Lyapunov function.

The estimated gains are used in defining a so-called gain automaton that forms the basis of an algorithmic criterion for the stability of the hybrid automaton. The associated gain automaton provides a systematic tool to detect potential sources of instability as well as an indication on to how to stabilize the hybrid systems by requiring appropriate delays for specific transitions.
\end{abstract}

\section{INTRODUCTION}

The hybrid automata model [2], [7] can be seen as an extension of the timed automata model [3]. A hybrid automaton is an automaton with locations and transitions (or switches) between the transitions, together with continuous dynamics in the locations, usually described by differential equations, and constraints on both locations and transitions. This model is a prominent model for the study of hybrid systems [19].

An important and interesting problem is that of the stability of a hybrid system. Even in the case of switched linear systems with asymptotically stable dynamics in each location, it is possible that the global behavior of the system is unstable (see e.g. [4]). For an overview of results on hybrid stability see [6], [12], [14]. Some results assume arbitrary switching between locations [14], [20]; it is then possible to look for a Lyapunov function common to all locations [1], [12]. The arbitrary switching assumption would be unsuitable in general for hybrid automata, since there the possible switchings are restricted by the guards of the transitions and the invariants of the locations.

Another stability criterion is that of multiple Lyapunov functions [4], [5], [12]. Each location is assumed to have a Lyapunov function. Now all behaviors of the system should satisfy the so-called non-increasing sequence property: if a location is visited again, the value of the Lyapunov function should be less than what it was at the previous time the location was visited. This is a sufficient condition for the stability of a hybrid automaton. In general checking the nonincreasing sequence property may be difficult, as checking all possible behaviors of a hybrid system is clearly not an option.

Another approach is the construction of Lyapunov functions that are either piecewise linear [11] or piecewise quadratic [10], [15], [17]. In the latter case the piecewise quadratic function should be continuous on the switching boundaries, which can be checked efficiently by solving a linear matrix inequality. The approach has originally been formulated for piecewise affine systems, where the state space is divided into regions, and to each region corresponds a dynamics. It is not so easy to adapt the approach to the more general model of hybrid automata.

In this paper we present an alternative approach that still makes use of the existence of a Lyapunov function in each location, but in addition incorporates the underlying automaton structure of the system model. Moreover, we demonstrate how a potentially unstable system may be stabilized by introducing appropriate transition delays.

The remainder of the paper is organized as follows. Section II gives basic definitions about hybrid automata and stability, and defines the class of hybrid automata used in this paper. Section III shows how to calculate an estimate of the gain using Lyapunov functions. It is shown how an optimal Lyapunov function can be chosen. Section IV shows how to use gain estimates in constructing an automaton that is used in an algorithm that detects non-contractive cycles in hybrid automata. The absence of such non-contractive cycles is a sufficient condition for the stability of the hybrid automaton. The occurrence, however, of non-contractive cycles does not imply instability of the hybrid system, rather it indicates that no simple guarantees for stability can be given. Due to the complex interaction between discrete switching between locations and continuous dynamics within location, a hybrid system having non-contractive cycles may be considered unsafe if stability is crucial. Section $\mathrm{V}$ describes how a cycle that is detected to be non-contractive may be stabilized by sufficiently delaying transitions belonging to that cycle. Then, in Section VI we introduce the notion of gain automaton. This is an automaton that is associated to the 
hybrid system under consideration and is used to detect, in a systematic way, non-contractive cycles. We present two algorithms: a passive detection algorithm, and an algorithm that not only detects non-contractive cycles, but also has the built-in option to determine how specific transitions in the cycle should be delayed such that the delayed cycle becomes contractive. Finally, in Section VII, we give conclusions and directions for future research. For basic notions of systems theory used in this paper we refer to [18].

\section{HYBRID AUTOMATA AND STABILITY}

The hybrid automaton model [2], [7] extends the classical notion of automaton by incorporating continuous dynamics in the locations, together with constraints at both locations and transitions.

Definition II.1. A hybrid automaton is a tuple $H=$ ( $X, L$, Init, Inv $, f, E$, Guard, Assign, $\Sigma)$ where:

- $X \subseteq \mathbb{R}^{n}$ is the continuous state space ranged over by the state vector $x$.

- $L$ is a finite set of locations.

- Init $\subseteq L \times \mathbb{R}^{n}$ is a set of initial location state pairs.

- Inv $: L \rightarrow 2^{X}$ assigns to each location $\ell$ an invariant to be satisfied by state $x$ while in location $\ell$.

- $f: L \rightarrow\left(X \rightarrow \mathbb{R}^{n}\right)$ assigns to each location $\ell$ a continuous vector field $f_{\ell}$ such that the state $x$ should satisfy $\frac{d}{d t} x=f_{\ell}(x)$.

- $E \subseteq L \times L$ is the set of transitions, also called switches.

- Guard : $E \rightarrow 2^{X}$ assigns to each transition a guard that has to be satisfied by state $x$ if the transition is taken.

- Assign $: E \rightarrow(X \rightarrow X)$ assigns to each transition an assignment that may alter state $x$ when the transition is taken.

- $\Sigma$ a set of transition labels. We assume a labeling function $l a b: E \rightarrow \Sigma$ and refer to transitions by their labels (assuming uniqueness).

In this paper we make a few additional assumptions:

- We assume that Init $=L^{\prime} \times \mathbb{R}^{n}$ for a set $L^{\prime} \subseteq L$ of initial locations, so that for a given initial location we can start with any state (which is technically convenient when studying stability).

- We assume that there are no invariants, i.e., Inv maps each location to the trivial condition true. This means that transitions are never forced, and it is possible to remain in a location forever.

- we assume that the dynamics in each location is linear, so $\frac{d}{d t} x=f_{\ell}(x)=A_{l} x, A_{\ell} \in \mathbb{R}^{n \times n}$.

- We assume that for each transition $e$ the guard is a hyperplane defined by an equation of the form $v_{e}^{\mathrm{T}} x=0$ for some $v_{e} \in \mathbb{R}^{n}$.

- We assume that the state is left unchanged by transitions (also called continuous switching), so for each transition $e, \operatorname{Assign}_{e}(x)=x$.

We call a hybrid automaton that satisfies these assumptions a Linear Continuous Hyperplane (LCH) hybrid automaton.
Example II.2. Consider the hybrid automaton consisting of four locations, $\ell_{1}, \ldots, \ell_{4}$. The dynamics in location $\ell_{i}$ is given by $\frac{d}{d t} x=A_{\ell_{i}} x, A_{\ell_{i}} \in \mathbb{R}^{2 \times 2}$, $i=1, \ldots 4$. The following events can occur: $E=$ $\left\{\left(\ell_{1}, \ell_{2}\right),\left(\ell_{1}, \ell_{4}\right),\left(\ell_{2}, \ell_{1}\right),\left(\ell_{2}, \ell_{3}\right),\left(\ell_{3}, \ell_{4}\right),\left(\ell_{4}, \ell_{2}\right)\right\}$, to which correspond labels $a$ to $f$ respectively. To each event there corresponds a switching line $L_{i j}$. For instance if the automaton is in location $\ell_{2}$ there are two possible transitions: to $\ell_{1}$ and to $\ell_{3}$. The transition to $\ell_{1}$ is enabled if and only if $x \in L_{21}$, whereas the transition to $\ell_{3}$ is possible when $x \in L_{23}$.

Definition II.3. A hybrid trace of an LCH hybrid automaton is a finite or infinite sequence of the form $\sigma=$ $x_{1} e_{1} x_{2} e_{2} \ldots x_{m-1} e_{m-1} x_{m}$, with an associated monotonically increasing timing sequence $\tau_{0} \tau_{1} \ldots \tau_{m}$ (with $\tau_{0}=0$, $\tau_{i} \in \mathbb{R} \cup\{\infty\}$ ), such that

- each $e_{i}$ is a transition from location $\ell_{i}$ to location $\ell_{i+1}$

- each $x_{i}$ is a mapping from $\left[\tau_{i-1}, \tau_{i}\right]$ to $\mathbb{R}^{n}$ satisfying $\frac{d}{d t} x_{i}=A_{\ell_{i}} x_{i}$

- initial and switching constraints and assignments are respected, so $\left(\ell_{1}, x_{1}(0)\right) \in$ Init, and for all $1 \leq i \leq$ $m-1: v_{e_{i}}^{\mathrm{T}} x_{i}\left(\tau_{i}\right)=0$ and $x_{i}\left(\tau_{i}\right)=x_{i+1}\left(\tau_{i}\right)$.

Remark II.4. To avoid Zeno behavior we impose that all transitions are delayed by a fixed minimal dwell time. This implies in particular that for infinite traces there holds $\lim _{i \rightarrow \infty} \tau_{i}=\infty$.

Definition II.5. An LCH hybrid automaton is stable iff $\forall \epsilon>$ $0 \exists \delta>0:\left\|x^{0}\right\|<\delta \Rightarrow$ for all hybrid traces $x_{1} e_{1} x_{2} e_{2} \ldots$ with $x_{1}(0)=x^{0}$ and $\forall i \forall t \in\left[\tau_{i-1}, \tau_{i}\right]:\left\|x_{i}(t)\right\|<\epsilon$. An automaton that is not stable is called unstable.

It is well known that even if for each location $\ell$ the dynamics is asymptotically stable, so the matrix $A_{\ell}$ is Hurwitz (i.e., all eigenvalues have negative real part, see [18]), still the hybrid automaton can be unstable (see e.g. [4] for a simple example). We say that a hybrid automaton has stable locations (with some abuse of terminology) if for each location $\ell$ the matrix $A_{\ell}$ is Hurwitz.

Now, our problem setting consists of two parts. Firstly, find sufficient conditions for the stability of an LCH hybrid automaton with stable locations. Secondly, if the sufficient conditions are not met, then provide systematic and quantified guidelines as to which transitions should be delayed to ensure stability.

\section{ESTIMATE OF GAINS VIA LYAPUNOV FUNCTIONS}

Suppose a location $\ell$ is entered via a transition $a$ with a state vector $x_{a}$ and is left via a transition $b$ with a state vector $x_{b}$. An indication as to how the location contributes to the stability or instability is the ratio of the norm of the outbound state and the inbound state. A ratio below one is in favor of stability whereas a ratio above one points at instability. Of course, since the ratio depends on the actual trace and state trajectory it does not provide a feasible stability indicator. Therefore we propose to use an 


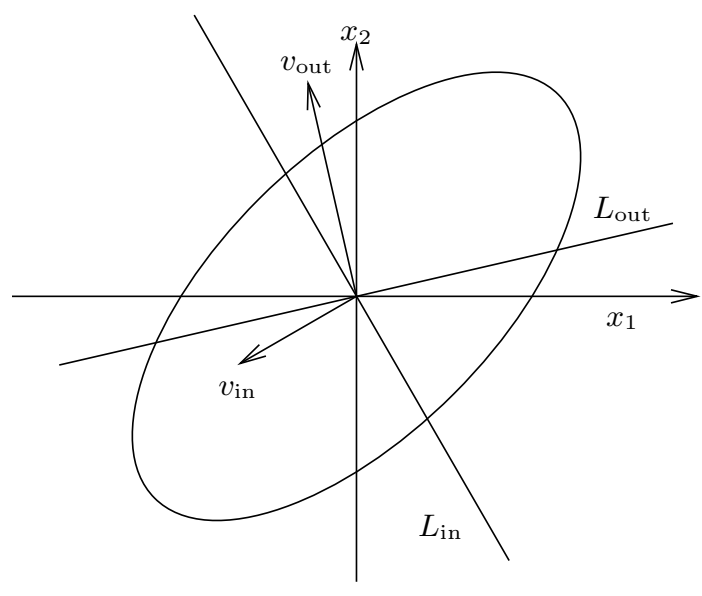

Fig. 1. Switching lines in a location

upperbound that only depends on the particular location and its corresponding inbound and outbound transitions.

Definition III.1. Consider an $\mathrm{LCH}$ hybrid automaton $H$ with Hurwitz locations. With each location $\ell$ we associate a symmetric positive definite matrix $P_{\ell}$ such that $A_{\ell}^{\mathrm{T}} P_{\ell}+$ $P_{\ell} A_{\ell} \leq 0$. Let $e_{\text {in }}$ represent a transition to $\ell$ and $e_{\text {out }}$ a transition from $\ell$ and let $L_{\mathrm{in}}$, given by $v_{\mathrm{in}}^{\mathrm{T}} x=0$, and $L_{\text {out }}$, given by $v_{\text {out }}^{\mathrm{T}} x=0$, denote the corresponding switching hyperplanes. Define ellipsoids $E_{\text {in }}$ and $E_{\text {out }}$ as $E_{\text {in }}=\{x \in$ $\left.L_{\text {in }} \mid x^{\mathrm{T}} P_{\ell} x=1\right\}$ and $E_{\text {out }}=\left\{x \in L_{\text {out }} \mid x^{\mathrm{T}} P_{\ell} x=1\right\}$. The corresponding gain $\alpha_{\text {in/out }}$ is defined as

$$
\alpha_{\text {in/out }}=\max _{x_{\mathrm{i}} \in E_{\mathrm{in}}, x_{\mathrm{o}} \in E_{\text {out }}} \frac{x_{\mathrm{o}}^{\mathrm{T}} x_{\mathrm{o}}}{x_{\mathrm{i}}^{\mathrm{T}} x_{\mathrm{i}}}
$$

Obviously, since $V(x)=x^{\mathrm{T}} P_{\ell} x$ is a Lyapunov function for $\frac{d}{d t} x=A_{\ell} x$ we have that any trajectory that enters the location through $L_{\text {in }}$ and leaves it through $L_{\text {out }}$ has the property that the ratio of the norms of outbound and inbound states is upperbounded by $\sqrt{\alpha_{\text {in }} \text { out }}$.

Two questions arise. Firstly, given $P_{\ell}$ how can we calculate $\alpha_{\text {in/out }}$ ? Secondly, it appears that the choice of $P_{\ell}$ affects $\alpha_{\text {in/out }}$. How to choose $P_{\ell}$ such that $\alpha_{\text {in/out }}$ is minimal? The latter question is treated in Section III-B.

\section{A. Calculation of the gains}

Let us explain how the calculation for given $P_{\ell}$ works. For ease of exposition we treat the two dimensional case first, see Figure 1.

The switching lines are given by $v_{\text {in }}^{\mathrm{T}} x=0$ and $v_{\text {out }}^{\mathrm{T}} x=0$ respectively. Let $\tilde{v}_{\text {in }}$ and $\tilde{v}_{\text {out }}$ be orthogonal to $v_{\text {in }}$ and $v_{\text {out }}$ respectively. Then it is not difficult to verify that

$$
\alpha_{\text {in } / \text { out }}=\frac{\tilde{v}_{\text {out }}^{\mathrm{T}} \tilde{v}_{\text {out }}}{\tilde{v}_{\text {in }}^{\mathrm{T}} \tilde{v}_{\text {in }}} \frac{\tilde{v}_{\text {in }}^{\mathrm{T}} P_{\ell} \tilde{v}_{\text {in }}}{\tilde{v}_{\text {out }}^{\mathrm{T}} P_{\ell} \tilde{v}_{\text {out }}} .
$$

If we choose $\tilde{v}_{\text {in }}$ and $\tilde{v}_{\text {out }}$ both on the same level curve, then (2) reduces to $\frac{\tilde{v}_{\text {out }}^{\mathrm{T}} \tilde{v}_{\text {out }}}{\tilde{v}_{\text {in }}^{\mathrm{T}} \tilde{v}_{\text {in }}}$.

For dimensions $n>2$ the situation is a bit more complicated since the maximization in (2) comes into play.
First notice that

$$
\alpha_{\text {in } / \text { out }}=\frac{\max _{x_{\mathrm{o}} \in E_{\mathrm{out}}} x_{\mathrm{o}}^{\mathrm{T}} x_{\mathrm{o}}}{\min _{x_{\mathrm{i}} \in E_{\text {in }}} x_{\mathrm{i}}^{\mathrm{T}} x_{\mathrm{i}}} .
$$

Next, e.g., the numerator of (3) can easily be calculated as follows. First assume that $v_{\text {out }}$ has norm one (otherwise normalize), then determine an orthogonal matrix $V_{\text {out }}$ such that the first column of $V_{\text {out }}$ is $v_{\text {out }}$. Define $P_{\text {out }}=V_{\text {out }}^{\mathrm{T}} P_{\ell} V_{\text {out }}$ and $\tilde{E}_{\text {out }}=\left\{z \in \mathbb{R}^{n} \mid z_{1}=0, z^{\mathrm{T}} P_{\text {out }} z=1\right\}$. Then

$$
\max _{x \in E_{\text {out }}} x^{\mathrm{T}} x=\max _{z \in \tilde{E}_{\text {out }}} z^{\mathrm{T}} z=\frac{1}{\lambda_{\min }\left(\tilde{P}_{\text {out }}\right)},
$$

where $\tilde{P}_{\text {out }}$ is obtained from $P_{\text {out }}$ by deleting the first row and and the first column. Furthermore $\lambda_{\min }(P)$ denotes the smallest eigenvalue of matrix $P$. In a similar way the denominator of (3) is obtained, resulting in

$$
\alpha_{\text {in } / \text { out }}=\frac{\lambda_{\max }\left(\tilde{P}_{\text {in }}\right)}{\lambda_{\min }\left(\tilde{P}_{\text {out }}\right)} .
$$

Remark III.2. For $n \geq 3$ two hyperplanes always intersect and hence also the sets $E_{\text {out }}$ and $E_{\text {in }}$ intersect. As a consequence the gain $\alpha_{\text {in } / \text { out }} \geq 1$.

\section{B. Optimizing the choice of Lyapunov function}

The gains that provide a stability indicator obviously depend on the Lyapunov functions in each location. Loosely, the fit of the level curves with respect to the trajectories determines the amount of conservatism. The better the fit, the less conservative the gain. Since Lyapunov functions are not unique, this suggests that we might be able to choose in each location a Lyapunov function that is optimal with respect to the switching planes. In this section we explain how this can indeed be done. We confine ourselves to quadratic Lyapunov functions. We show that for a given stable matrix $A$ the set of quadratic Lyapunov functions is convex and compact. Furthermore the stability gain corresponding to $A$ and given switching hyperplanes depends continuously on the Lyapunov function so that at least the optimum exists. We illustrate the effectiveness of these results by means of a two dimensional example.

Let $A \in \mathbb{R}^{n \times n}$ be a stable matrix. We are interested in the set of quadratic Lyapunov functions, or, more precisely, the set of level curves corresponding to quadratic Lyapunov functions. To enable the comparison of different Lyapunov functions we choose a nonzero $x_{0} \in \mathbb{R}^{n}$ and define the set

$$
\Omega_{x_{0}}=\left\{P \in \mathbb{R}^{n \times n} \mid A^{\mathrm{T}} P+P A \leq 0, x_{0}^{\mathrm{T}} P x_{0}=1\right\} .
$$

In fact, $\Omega_{x_{0}}$ is a parametrization of the level curves corresponding to quadratic Lyapunov functions and level unity.

Lemma III.3. Let $A \in \mathbb{R}^{n \times n}$ and let $x_{0} \in \mathbb{R}$ be a nonzero vector that does not belong to an A-invariant subspace of dimension at most $n-1$. Let $U$ be an open neighborhood of $0 \in \mathbb{R}$. Then $\operatorname{span}_{t \in U}(\exp (A t)) x_{0}=\mathbb{R}^{n}$.

Theorem III.4. Let $A \in \mathbb{R}^{n \times n}$ be a stable matrix and let $x_{0} \in \mathbb{R}$ be a nonzero vector. Define $\Omega=\left\{P \in \mathbb{R}^{n \times n} \mid\right.$ $A^{T} P+P A \leq 0$ and $\left.x_{0}^{T} P x_{0}=1\right\}$. 
1. If $x_{0}$ does not belong to a proper A-invariant subspace then $\Omega$ is compact.

2. Every $P \in \Omega$ is positive semi-definite.

3. $\Omega$ is convex.

Example III.5. Let the dynamics in a given location be given by $\frac{d}{d t} x=A x$ with

$$
A=\left[\begin{array}{cc}
0 & 1 \\
-2 & -3
\end{array}\right]
$$

The switching lines are given by

$$
L_{\text {in }}=\lambda \underbrace{\left[\begin{array}{l}
0 \\
1
\end{array}\right]}_{a_{\text {in }}} \quad L_{\text {out }}=\lambda \underbrace{\left[\begin{array}{l}
1 \\
0
\end{array}\right]}_{a_{\text {out }}}
$$

As explained in Section III-A, for a given Lyapunov function $V(x)=x^{\mathrm{T}} P x$ the gain is defined as

$$
\alpha_{P}=\frac{a_{\mathrm{out}}^{\mathrm{T}} a_{\mathrm{out}}}{a_{\text {in }}^{\mathrm{T}} a_{\text {in }}} \frac{a_{\text {in }}^{\mathrm{T}} P a_{\text {in }}}{a_{\text {out }}^{\mathrm{T}} P a_{\mathrm{out}}}=\frac{p_{22}}{p_{11}}
$$

The set $\Omega_{a_{\text {in }}}$ is depicted in Figure 2. To find the optimal

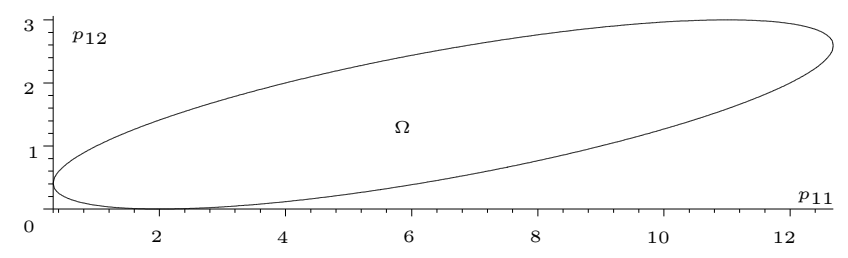

Fig. 2. The set $\Omega_{a_{\text {in }}}$ of all Lyapunov functions.

Lyapunov function we want to minimize $\alpha_{P}$ over $\Omega_{a_{\text {in }}}$. For this example this amounts to the minimization of $\frac{1}{p_{11}}$ or, equivalently, the maximization of $p_{11}$. Theorem III.4 guarantees the optimum exists. Using Maple we found two extreme Lyapunov functions. One that minimizes $\alpha_{P}$ and one that maximizes $\alpha_{P}$ :

$$
P_{\min }=\left[\begin{array}{cc}
12.70 & 2.59 \\
2.59 & 1
\end{array}\right] \quad P_{\max }=\left[\begin{array}{cc}
.32 & .41 \\
.41 & 1
\end{array}\right]
$$

The corresponding minimum and maximum values of the gains are

$$
\alpha_{\min } \approx 0.38 \quad \alpha_{\max } \approx 2.61
$$

In Figure 3 the level curves of the two Lyapunov functions are drawn together with the phase portrait of $\frac{d}{d t} x=A x$. This clearly shows the benefit of using the non-uniqueness of Lyapunov function. The one that minimizes $\alpha$ is obviously dramatically less conservative than the one that maximizes $\alpha$.

Remark III.6. In higher dimensions the minimization of the gain may be cumbersome to perform. Indeed, the expression in (5) is a non-convex function of $P$. Notice, however, that $\lambda_{\max }(P)$ is convex and $\lambda_{\min }(P)$ is concave. A suboptimal solution is therefore obtained by disregarding either the numerator or the denominator in (5).

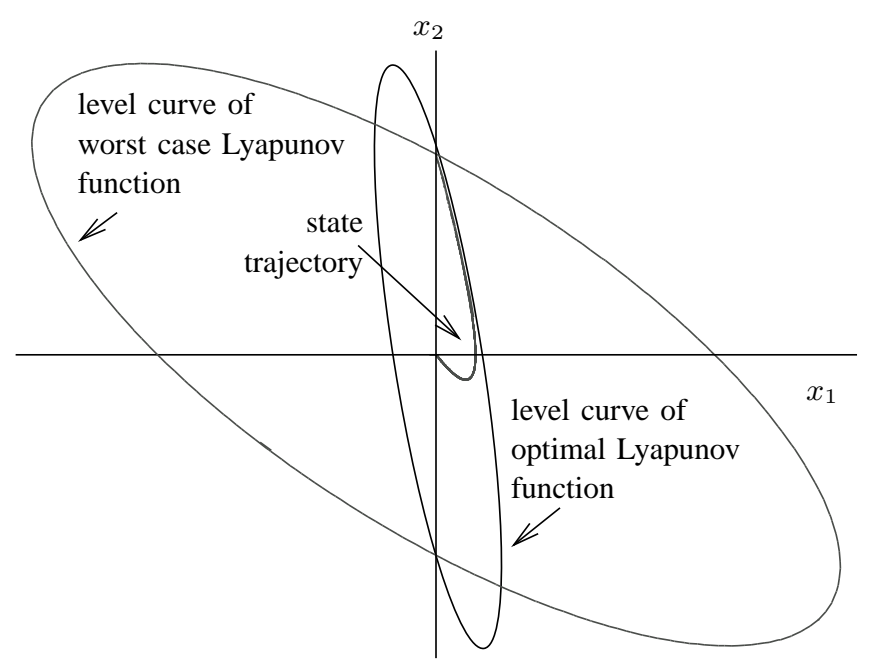

Fig. 3. Level curves of the extreme Lyapunov functions

\section{NON-CONTRACTIVE CYCLES}

Suppose we have a hybrid automaton with Hurwitz locations. If for each location that can be visited more than once the estimated gain is $\leq 1$, then it can be seen that the hybrid automaton is stable: since the number of locations in a trace that are visited only once (seen as a function of a trace) is bounded, there is a bound to the gains corresponding to the traces. However, such a condition is unnecessarily restrictive as it does not take into account situations where a higher gain in one location is compensated by a lower gain in another location. So we need a more sophisticated condition.

Definition IV.1. Let $H$ be a hybrid automaton, then a contractive cycle of $H$ is a sequence of transitions $C=$ $e_{1} e_{2} \ldots e_{m}$ such that each $e_{i}$ is a transition from $\ell_{i}$ to $\ell_{i+1}$, with $\ell_{1}=\ell_{m+1}$, and $\gamma_{C}=\alpha_{e_{1} e_{2}} \cdot \alpha_{e_{2} e_{3}} \cdot \ldots \cdot \alpha_{e_{m} e_{1}} \leq 1$. The scalar $\gamma_{C}$ is called the cycle gain.

Theorem IV.2. Let $H$ be an LCH hybrid automaton with Hurwitz locations. Then: $H$ is unstable $\Rightarrow H$ has a noncontractive cycle.

\section{StABILIZING NON-CONTRACTIVE CYCLES BY DELAYING TRANSITIONS}

If a cycle is non-contractive, that is, the product of the gains exceeds one, then the cycle may still be stabilized by appropriately delaying transitions. Indeed, since all locations have asymptotically stable dynamics, sufficiently delaying transitions reduce all separate gains to a value not exceeding unity. This, however, is not necessary. It suffices to delay one or more transitions in the cycle such that the cycle becomes contractive, that is, such that the product of all gains does not exceed one. How to exactly achieve this may be quantified through the following lemma.

Lemma V.1. Let $A$ be a Hurwitz matrix, $Q=Q^{T}$ is a positive definite matrix and let $P$ satisfy the associated 
Lyapunov equation $A^{T} P+P A=-Q$. Furthermore let $x(t)$ be the solution of $\frac{d}{d t} x(t)=A x(t)$ with $x(0)=x_{0}$. Then

$$
x(t)^{T} P x(t) \leq x_{0}^{T} P x_{0} \mathrm{e}^{-\lambda t},
$$

where $\lambda=\sigma_{\min }\left(P^{-\frac{1}{2}} Q P^{-\frac{1}{2}}\right)$.

Next we describe how to use Lemma V.1 to stabilize a non-contractive cycle. Let $C$ be a non-contractive cycle. This means that the product of the estimated gains is larger than one. Let $\alpha$ be an estimated gain corresponding to location $\ell$ belong to the cycle $C$. Denote the dynamics in $\ell$ by $\frac{d}{d t} x=A_{\ell} x$ and let $A^{T} P+P A=-Q$ be the corresponding Lyapunov equation on the basis of which $\alpha$ has been calculated. This may be the optimized $P$ obtained as described in Section III-B provided it is positive definite. However if that $P$ is positive semi-definite, then the gain estimate has to be recalculated on the basis of positive definite $P$ followed by the calculation of the delay.

As noted, the interpretation of the transition gain $\alpha$ is that it reflects an upper bound on the state gain corresponding to the event "enter $\ell$ followed by 'leave $\ell$. The gain $\alpha$ is based on level sets of the Lyapunov function $x^{T} P x$ only, time evolution is not taken into account. In that sense $\alpha$ may be somewhat conservative. On the other hand, it would be very difficult to even estimate how long we stay in $\ell$. However, by imposing a minimum delay it becomes feasible to estimate the reduction in gain estimate.

More precisely: Let $H$ be a hybrid automaton and let $C$ be cycle of $H$, i.e., a sequence of transitions $e_{1} e_{2} \ldots e_{m}$ such that each $e_{i}$ is a transition from $\ell_{i}$ to $\ell_{i}$, with $\ell_{1}=\ell_{m+1}$, and let $\alpha_{e_{1} e_{2}} \cdot \alpha_{e_{2} e_{3}} \cdot \ldots \cdot \alpha_{e_{m} e_{1}}$ be the corresponding gain estimates. Denote by $\delta_{e_{i-1}, e_{i}} \geq 0$ the delay corresponding to the transition pair $\left(e_{i-1}, e_{i}\right)$. The interpretation of $\delta_{e_{i-1} e_{i}}$ is that after transition $e_{i-1}$ a delay of at least $\delta_{e_{i-1} e_{i}}$ has to be respected before transition $e_{i}$ may be taken. In this way $\delta_{e_{i-1} e_{i}}$ acts as a kind of guard. During the delay $\delta_{e_{i-1} e_{i}}$ location $\ell_{i}$ is active. The delayed cycle gain is now defined as:

$$
\gamma_{C}(\delta)=\prod_{i=1}^{m+1} \alpha_{i} \mathrm{e}^{-\lambda_{i} \delta e_{i-1} e_{i}} .
$$

It now suffices to choose the delays $\delta_{e_{i-1} e_{i}} \mathrm{~s}$ such that the cycle gain becomes smaller than or equal to one.

The above is, of course, closely related to the concept of dwell-time as introduced studied in [8], [16]. However, there dwell times are defined in terms of the dynamics in locations as isolated entities. In our context delays are studied in relation to all transitions in a given cycle.

\section{GAin AUTOMATA AND DETECTION OF NON-CONTRACTIVE CYCLES}

Theorem IV.2 together with the transition delays provides us with a sufficient condition for stability, namely the absence of non-contractive cycles. In order to check for noncontractive cycles we first transform a hybrid automaton into what we call gain automaton.

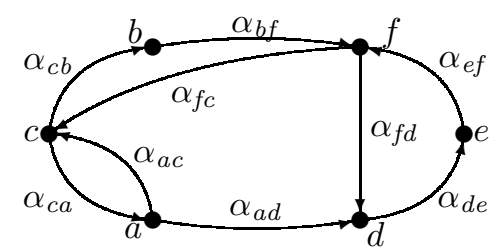

Fig. 4. Example of a gain automaton

Definition VI.1. A gain automaton is a tuple $G A=$ $\left(S, S^{0}, G\right)$ where

- $S$ is the set of nodes,

- $S^{0}$ is the set of initial nodes,

- $G \subseteq S \times \mathbb{R}^{+} \times S$ is the set of edges labeled with gains.

Definition VI.2. Let $H$ be a hybrid automaton, then the gain automaton for $H$ is defined by $G A(H)=\left(S_{H}, S_{H}^{0}, G_{H}\right)$ where

- The nodes of the gain automaton are the transitions of $H$, so $S_{H}=E$.

- The initial nodes $S_{H}^{0}$ are the transitions from an initial location of $H$.

- For each pair of transitions $e, e^{\prime}$ in $H$ such that $\stackrel{e}{\longrightarrow}$ $l \stackrel{e^{\prime}}{\longrightarrow}$ there is an edge $e \stackrel{\alpha_{e e^{\prime}}}{\longrightarrow} e^{\prime}$ in $G_{H}$.

Example VI.3. Let $H$ be the hybrid automata of Example II.2, then the gain automaton $G A(H)$ is given in Figure 4.

We define an algorithm on the gain automaton of a hybrid automaton for the detection of non-contractive cycles. This algorithm is inspired by the well-known algorithm for transforming an automaton into an equivalent regular expression (see e.g. [9], [13]). It works by successively deleting nodes of the gain automaton, while transforming the edges. The basic steps of the algorithm are:

- Node elimination: a node is eliminated. Each possible pair of an incoming and outgoing edge of this node leads to a new edge, labeled with the product of the gains, as illustrated in Figure 5(a).

- Double edge elimination: if two edges have the same initial and final node they are transformed into a single edge, labeled with the maximum of the gains, as illustrated in Figure 5(c).

- Loop edge analysis: it is possible that deleting a node creates a loop edge, as illustrated in Figure 5(b). If the gain of such a loop edge is $>1$ (we call this a non-contractive loop edge) the algorithm is terminated. Otherwise, the loop edge is removed.

Algorithm VI.4. Input: a gain automaton $G A$. check all loop edges;

if a non-contractive loop edge is found

then terminate;

remove all loop edges;

repeat

eliminate a state;

eliminate all resulting double edges; 


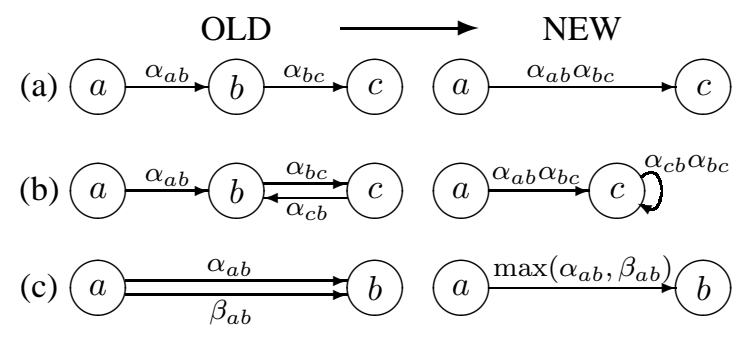

Fig. 5. Basic steps of the algorithm

analyze all resulting loop edges;

if a non-contractive loop edge is found

then terminate;

remove all loop edges

until there is only one state

Theorem VI.5. Let $H$ be an LCH hybrid automaton with Hurwitz locations. Then: Algorithm VI.4 detects a noncontractive loop edge in $G A(H) \Leftrightarrow H$ contains a noncontractive cycle.

Of course, detecting a non-contractive cycle may not be the end of the story. In case transition delay is feasible noncontractive cycles may be stabilized by delay as detailed in Section V. The algorithm VI.4 then becomes:

Algorithm VI.6. Input: a gain automaton $G A$. check all loop edges;

if a non-contractive loop edge is found

then terminate;

remove all loop edges;

repeat

\author{
eliminate a state; \\ eliminate all resulting double edges; \\ analyze all resulting loop edges; \\ if a non-contractive loop edge is found \\ then stabilize by delay; \\ remove all loop edges
}

until there is only one state

Algorithm VI.6 provides a systematic means to detect potential unstable cycles and subsequently stabilizing these.

The number of nodes in $G A(H)$ is quadratic in the number of nodes of $H$, and the complexity of Algorithm VI.4 and VI.6 is linear in the number of nodes of $G A$, so the complexity of Algorithm VI.4 and VI.6 is quadratic in the number of nodes of $H$. This means we have a computationally efficient way of checking the sufficiency condition for stability, viz. the absence of non-contractive cycles.

\section{CONCLUSIONS}

We have derived a sufficient condition for the stability of a hybrid automaton, viz. the absence of non-contractive cycles, together with an algorithm for efficiently checking this condition. In addition, we have proposed a systematic method through which transition delays may be introduced to stabilize potentially unstable cycles. In principle the incorporation of the delays could be part of the criterion according to which the optimal Lyapunov function is constructed. At this moment, however, this appears to yield an unfeasible optimization problem.

We have made use of both systems theoretic concepts (in calculating the estimated gains) and computer science concepts (in checking the cycles in the gain automaton), thereby doing justice to both the continuous and the discrete aspects of hybrid systems.

\section{REFERENCES}

[1] Andrei A. Agrachev and Daniel Liberzon. Lie-algebraic stability criteria for switched systems. SIAM Jour. Ctr. Opt., 40:253-269, 2001.

[2] Alur, R., C. Courcoubetis, T.A. Henzinger, and P.-H. Ho. Hybrid automata: an algorithmic approach to the specification and verification of hybrid systems. In R.L. Grossman, A. Nerode, A.P. Ravn, and H. Rischel, editors, Hybrid Systems I, volume 736 of Lecture Notes in Computer Science, pages 209-229. Springer Verlag, 1993.

[3] Rajeev Alur and David L. Dill. A theory of timed automata. Theoretical Computer Science, 126:183-235, 1994.

[4] Michael S. Branicky. Stability of switched and hybrid systems. In Proc. 33rd IEEE Conf. Decision and Control, pages 3498-3503, Orlando, FL, 1994.

[5] Michael S. Branicky. Multiple Lyapunov functions and other analysis tools for switched and hybrid systems. IEEE Trans. Aut. Contr. 43:475-482, 1998.

[6] DeCarlo, Raymond A., Michael S. Branicky, Stefan Pettersson, and Bengt Lennartson. Perspectives and results on the stability and stabilizability of hybrid systems. In Proc. of the IEEE, volume 88, pages 1069-1082, 2000.

[7] Thomas A. Henzinger. The theory of hybrid automata. In Proceedings LICS'96, pages 278-292, 1996.

[8] J.P. Hespanha and S.A. Morse. Stability of switched systems with average dwell-time. In Proc. 38th IEEE Conf. Decision and Control, pages 2655-2660, Phoenix, AZ, 1999.

[9] Rajeev Motwani Hopcroft, John E. and Jeffrey D. Ullman. Introduction to Automata Theory, Languages, and Computation. AdissonWesley, second edition, 2001.

[10] Mikael Johansson and Anders Rantzer. Computation of piecewise quadratic Lyapunov functions for hybrid systems. IEEE Trans. Aut. Contr., 43:555-559, 1998.

[11] Xenofon D. Koutsoukos and Panos J. Antsaklis. Design of stabilizing switching control laws for discrete and continuous-time linear systems using piecewise-linear Lyapunov functions. Technical Report ISIS Technical Report ISIS-2001-002, ISIS, 2001.

[12] Daniel Liberzon and A. Stephen Morse. Basic problems in stability and design of switched systems. IEEE Control Systems Magazine, 19:59-70, 1999.

[13] Peter Linz. An Introduction to Formal Languages and Automata. Jones and Bartlett publishers, third edition, 2001.

[14] Anthony N. Michel. Recent trends in the stability analysis of hybrid dynamical systems. IEEE Transactions on Circuits and Systems - I, 45:120-134, 1999.

[15] Giancarlo Ferrari-Trecate Mignone, Domenico and Manfred Morari. Stability and stabilization of piecewise affine and hybrid systems: an LMI approach. In Proceedings 39th IEEE CED, pages 504-509, 2000.

[16] A.S. Morse. Dwell-time switching. In Proceedings of the second European Control Conference, pages 176-181, Groningen, The Netherlands, 1993.

[17] Stefan Pettersson and Bengt Lennartson. Stability and robustness for hybrid systems. In Proc. 35th IEEE Conf. Decision and Control, pages 1202-1207, Kobe, Japan, 1996.

[18] J.W. Polderman and J.C. Willems. Introduction to mathematical systems theory: a behavioral approach, volume 26 of Texts in Applied Mathematics. Springer, New York NY, USA, 1998.

[19] A.J. van der Schaft and J.M. Schumacher. An Introduction to Hybrid Dynamical Systems, volume 251 of Lecture Notes in Control and Information Sciences. Springer-Verlag, London, 2000.

[20] Anthony N. Michel Ye, Hui and Ling Hou. Stability theory for hybrid dynamical systems. IEEE Trans. Aut. Contr., 43:461-474, 1998. 